\title{
Patient-reported involvement of the eighth cranial nerve in giant cell arteritis
}

\author{
Vadivelu Saravanan $^{1} \cdot$ Susan Pugmire ${ }^{1} \cdot$ Mavis Smith $^{1} \cdot$ Clive Kelly $^{1}$ (D) \\ Received: 26 June 2019 / Revised: 29 July 2019 / Accepted: 8 August 2019 / Published online: 16 August 2019 \\ (C) The Author(s) 2019
}

\begin{abstract}
Introduction The frequency of eighth nerve lesions in patients with giant cell arteritis (GCA) has rarely been examined. However, sudden onset deafness has been recorded as a presenting feature of GCA on several occasions. This study sought to establish how common this and other symptoms of eighth nerve involvement are in a large retrospective survey.

Methods We contacted 170 patients with GCA and 250 matched PMR patients, inviting them to participate in a questionnaire survey of symptoms of eighth nerve dysfunction. We compared the presence of deafness, tinnitus, loss of balance and vertigo in both groups and examined the relationship between the onset of these symptoms and other features of GCA.

Results A total of 317 patients were recruited. The percentage of patients with symptoms of possible vestibulocochlear disease prior to commencement of steroid therapy was significantly greater among GCA patients than PMR patients for all domains. Hearing loss which was twice as common in GCA as in PMR (53\% vs 26\%) $[p=0.001]$. Deafness was concurrent in $35 \%$ of GCA patients with other symptoms and $45 \%$ reported colocation with headache. Recovery with steroids occurred in $56 \%$ of these.

Conclusion Symptoms of eighth nerve dysfunction are present in over half of patients with GCA. Recovery with steroids was predicted by concurrence with headache in terms of both timing and location. It appears that eighth nerve involvement, especially acute hearing loss, is a not infrequent feature of GCA and often responds well to steroid therapy. Clinicians should enquire about these symptoms when evaluating a patient for possible GCA.
\end{abstract}

Key Points

- Deafness is a frequent presenting feature of giant cell arteritis.

- Vertigo, tinnitus and loss of balance are also often reported by GCA sufferers.

- Steroid therapy is more likely to relieve these symptoms if they are ipsilateral and concurrent with headache.

Keywords Deafness $\cdot$ Giant cell arteritis $\cdot$ Loss of balance $\cdot$ Vertigo $\cdot$ Vestibulocochlear nerve

\section{Introduction}

Giant cell arteritis (GCA) is a serious condition, with a prevalence of between 17 and 154 per 10,000 population in the UK $[1,2]$. Northern Europe has the highest reported prevalence and women are affected at least twice as often as men [3]. GCA is a systemic vasculitis of medium and large arteries often with ophthalmic involvement, including ischemic optic neuropathy, retinal artery occlusion and ocular motor cranial

Clive Kelly

cliveryton@gmail.com

1 Department of Rheumatology, Queen Elizabeth Hospital, Sheriff Hill, Gateshead, UK nerve palsies. GCA usually affects the superficial temporal arteries and the ophthalmic, occipital, vertebral, posterior ciliary and proximal vertebral arteries [2]. Common symptoms include temporal tenderness, jaw claudication and doublevision or occasionally loss of vision. Approximately 20\% of cases in total describe visual disturbances [3].

Cranial nerve palsies have been described in $2-15 \%$ of patients with GCA [4], although these rarely affect more than one cranial nerve. The third nerve is the most commonly affected, but involvement of the fourth, fifth, sixth and eighth nerves have also been reported [4]. Over the past 25 years, only $1.5 \%$ of all GCA cases have reported loss of hearing and/ or vertigo as a symptom [5]. However, in clinical practice, many patients report loss of hearing or tinnitus commensurate with the onset of GCA symptoms, and many patients describe 
at least partial resolution with the commencement of steroid treatment. By contrast vestibulo-auditory manifestations were quite common in a prospective study [6]. Common symptoms were unilateral or bilateral hearing loss, vertigo and tinnitus. In total, $64 \%$ had subjective hearing impairment, $52 \%$ had vertigo and $50 \%$ had tinnitus. These were reversible in most cases following steroid therapy [6].

Polymyalgia rheumatica (PMR) is a condition that causes muscle stiffness in patients around the neck and shoulders. It is rare under the age of 50 and usually affects patients over the age of 65 years. PMR is characterised by proximal muscle stiffness, usually in the morning, that lasts for more than $45 \mathrm{~min}$. It is estimated that 1 in every 1200 people in the UK will develop the condition annually [7]. Approximately one in five patients of these then develop GCA (NHS Choices 2017). Up to 50\% of patients with GCA also have PMR [8].

Despite the dearth of published evidence relating to symptoms such as loss of hearing and tinnitus in GCA, frequent reports by patients and the results of a single prospective survey [6] led us to conclude that it is appropriate to investigate the involvement of the vestibulocochlear nerve, as part of the possible spectrum of disease seen in GCA, using PMR without GCA patients as controls.

\section{Methods}

\section{Aims and objectives}

The study aimed to investigate the hypothesised connection between patient-reported symptoms of hearing loss and other potential symptoms of eighth nerve involvement with the onset of GCA. We aimed to gauge the frequency of such symptoms within the GCA patient population, using patients with PMR and no evidence of GCA as controls, and thereby deduce the frequency of involvement of the vestibulocochlear nerve in patients with GCA.

The objective was to survey 250 patients with GCA for symptoms of possible vestibulocochlear dysfunction and a further 250 age- and sex-matched control patients with PMR but no GCA. We intended to calculate the prevalence of each of four possible symptoms in both populations. Furthermore, we wished to assess the relationship between the site of any auditory symptoms (ipsilateral or bilateral) and the site of headache, together with the temporal relationship between them. We wanted to assess the response of all vestibulocochlear symptoms to steroid therapy and to assess the potential association between colocation (ipsilateral headache and deafness /tinnitus) and steroid responsiveness. We also wished to explore the relationship between concurrence (synchronous onset of headache and auditory symptoms) and steroid responsiveness. We hoped that this would allow patients and their clinicians to identify the disease in its fullest manifestations more rapidly, offering the potential to improve treatment outcomes.

The study was conducted via a Likert scale and narrative questionnaire. Participants were identified through the registered charity PMR-GCA UK Support Group who funded the study. The Charity holds a comprehensive database of patients, who have previously consented to be contacted for research activities.

\section{Inclusion and exclusion criteria}

Inclusion criteria were defined as:

- 50 years old or above, diagnosed with GCA (active) or PMR (controls)

- Contact information contained within the funders database

- Able and willing to complete a paper version questionnaire

Exclusion criteria were defined as:

- Under 50 years of age or not currently diagnosed with GCA or PMR

- Insufficient contact information contained within the funders database

- Unable to complete a paper questionnaire

\section{Recruitment}

A named member of the funders team (MS) identified patients and sent a Participant Information Sheet and Questionnaire via the post. A return addressed envelope was contained in the research pack. Completed questionnaires were returned to the Chief Investigator based at the Gateshead Health NHS Foundation Trust. This process has been tested and validated in the pilot phase of the study. The questionnaire shall not collect any identifiable patient data and for the protection of the participants, the Chief Investigator shall not have access to the funders database. All questionnaires were anonymised via a study number known only to the funders.

\section{Materials}

It was deemed most appropriate to conduct the questionnaire via paper-based postal distribution. Because of the significant age of the patients involved, this was felt to carry a higher likely response rate than an online questionnaire. Hence, potential participants were identified by the funder and a research pack sent in the post. The pack contained a participant Information Sheet, questionnaire and return addressed envelope. Patients wishing to participate in the study were given 
3 months to return their completed questionnaire to the Chief Investigator. All paper questionnaires are retained in a locked cabinet. All data are to be archived after the official close of the study for 5 years, as per local archiving policies.

\section{Data analysis}

Information from the questionnaires was collated into a password-protected Excel spreadsheet. It was held on an NHS Server only. Data entry was to be performed by a member of the study team (SP). Data were checked by the Chief Investigator for accuracy. Each questionnaire was provided with a unique identification study code on receipt.

It was not possible to contact participants to complete any blank fields. Therefore, it was only deemed acceptable if a questionnaire was completed in at least $75 \%$ of fields. Answers were analysed using a variety of descriptive statistics and narrative-based answers were analysed using qualitative approaches.

\section{Ethical issues and consent}

As a condition of inclusion within the database holding the details of potential participants, prior written consent to postal surveys had been obtained. Under these circumstances, it was deemed that a returned questionnaire would be taken as implied consent. This approach was approved by the Health Research Authority and Research Ethics Committee. The patient information sheet (PIS) explained that there was no direct benefit to participants, nor any perceived potential for harm. However, subsequent patients may benefit by earlier and more accurate diagnosis with the potential for more rapid and potentially effective therapeutic intervention.

\section{Statistics}

The study aimed to recruit 500 participants. It was estimated that this is feasible with a $75 \%$ response rate in comparison to the number of participants currently on the Charities database [9].

However, it is noted that the response rate may be greater or lower than expected. To ensure that sufficient respondents are included in the study, the margin of error is calculated dependent upon the number of respondents [10].

It is worth noting that "the minimum sample size needed to evaluate features with an acceptable tolerable error (relating to the studies research question and objectives, assuming a relative tolerable error of $5 \%$, a coefficient of variation $(\mathrm{C})$ of a population of $5 \%$ and a pairwise correlation coefficient $\mathrm{p}$ (rho) of 0.5 ) is 231 participants, who provide calculations in table format allowing for a 5-item Likert scale. However, due to the population being finite, the sample size must be adjusted [9]:

$n=\frac{n_{0} N}{n_{0}+(N-1)}=\frac{231 \times(\text { Unknown at present })}{231+(\text { Unknown }-1)}=? ?$

Comparisons between the GCA and PMR groups were made using Pearson's chi-square test because of the uneven numbers of returned questionnaires from within the two groups.

\section{Results}

The intention had been to recruit 250 patients with each of GCA and PMR but we failed to achieve this. Questionnaires were sent to only 170 patients with GCA, while they were delivered to 250 with PMR. This difference was due to the difficulty in identifying reliable diagnoses in sufficient patients. However, response rates between the two groups were very similar at $74 \%$ among patients with GCA and $76 \%$ for PMR patients. This led to a total of 317 patients recruited to the study. Of these, 128 had giant cell arteritis and 189 had polymyalgia rheumatica. Sufficient patients were recruited to meet the statistical requirements for significance.

The median age of patients at diagnosis did not differ between GCA and PMR (71 years vs 70 years). The percentage of male patients was also similar in both groups ( $8 \%$ vs $9 \%$ ). As expected, the median initial dose of prednisone was greater in patients with GCA than in those with PMR $(60 \mathrm{mg}$ vs $15 \mathrm{mg}$ ). The total duration of therapy with prednisone at the time of data collection was not significantly different between GCA and PMR patients (18 months vs 17 months), and $72 \%$ of patients in each group were still taking prednisone at the time of the study. These data are shown in Table 1.

The percentage of patients with symptoms of possible vestibulocochlear disease prior to commencement of steroid therapy was significantly greater among GCA patients when compared to those with PMR. This observation extended across all four symptom domains and is shown in Table 2. Specifically, patients with GCA reported hearing loss in over half of cases which were twice as often as reported by patients with PMR (53\% vs 26\%) $[p=0.001]$.

Tinnitus was also reported significantly more commonly among GCA patients than by those with PMR, although the difference was less marked (51\% vs 39\%) [ $p=0.02]$. Loss of balance was recorded by well over half of patients with GCA, and again this was significantly less frequent among those with PMR (57\% vs 38\%) [ $p=0.01]$. Finally, vertigo was the least frequently reported symptom but was still almost twice 
Table 1 To compare the demography of patients with giant cell arteritis (GCA) and polymyalgia rheumatica (PMR) at point of study entry

\begin{tabular}{|c|c|c|c|c|c|}
\hline & $\begin{array}{l}\text { Mean age } \\
\text { (years) }\end{array}$ & $\begin{array}{l}\text { Number }(N) \\
(\%) \text { male }\end{array}$ & $\begin{array}{l}\text { Duration of symptoms } \\
\text { (months) }\end{array}$ & $\begin{array}{l}\text { Initial dose of } \\
\text { Prednisone (mg) }\end{array}$ & $\begin{array}{l}N(\%) \text { still on } \\
\text { steroid }\end{array}$ \\
\hline GCA & 71 & $6(8)$ & 18 & 60 & $50(72)$ \\
\hline PMR & 70 & $6(9)$ & 17 & 15 & $50(72)$ \\
\hline$P$ value & - & - & - & 0.001 & - \\
\hline
\end{tabular}

as commonly reported among those with GCA than those with PMR (39\% vs 22\%) [ $p=0.003]$.

The data relating to the timing of potential vestibulocochlear symptoms with the rheumatological diagnosis was important. Thirty-five per cent of patients with GCA and hearing loss stated that their deafness was concurrent with the onset of other symptoms of GCA, and $45 \%$ said that this was ipsilateral and unilateral with headache. Importantly, recovery with steroids in line with other symptoms was reported by only $20 \%$ of GCA patients overall but was much higher at $56 \%$ among those with both concurrent symptoms and colocation. By comparison, patients with PMR who reported hearing loss had less symptom concurrence (12\%) and little recovery with steroids $(5 \%)$. Differences between GCA and PMR were significant $[p=0.01]$.

Among those with GCA and tinnitus, symptoms concurred with diagnosis in $33 \%$ and were purely ipsilateral with headache in $42 \%$ of cases. However, improvement with steroids was only reported in $14 \%$ overall. Again, those patients with both concurrent symptoms and ipsilateral location had a much higher steroid response rate of $42 \%$. Corresponding figures for those with PMR and tinnitus were $28 \%$ for symptom concurrence while recovery with steroid recovery was reported in just $9 \%$.

In those GCA patients who recorded loss of balance, this was reported to have occurred at the same time as other symptoms in $53 \%$ but improved with steroid therapy in only $27 \%$. Comparative figures for PMR patients were not significantly different at $45 \%$ and $17 \%$ respectively.

Finally, vertigo among patients with GCA was concurrent with other clinical features in $50 \%$ but recovered with steroids in just $17 \%$. By comparison, among PMR patients, $28 \%$ had symptom concurrence while $22 \%$ reported steroid-related

Table 2 To compare the prevalence of recorded vestibulocochlear symptoms between 128 patients with giant cell arteritis (GCA) and 189 with polymyalgia rheumatica (PMR) in numbers (percentages) of total

\begin{tabular}{llll}
\hline & GCA & PMR & $P$ value \\
\hline Hearing loss & $68(53 \%)$ & $49(26 \%)$ & 0.001 \\
Tinnitus & $65(51 \%)$ & $74(39 \%)$ & 0.02 \\
Loss of balance & $73(57 \%)$ & $72(38 \%)$ & 0.01 \\
Vertigo & $50(39 \%)$ & $41(22 \%)$ & 0.003 \\
\hline
\end{tabular}

recovery, but with poor correlation. Although vertigo was statistically more likely to be concurrent with other symptoms in GCA patients, the responsiveness of vertigo to steroid therapy did not differ significantly between GCA and PMR.

These data are summarised in Table 3.

\section{Discussion}

Cranial nerve palsies are well recognised as complications of GCA but these most commonly affect the nerves responsible for eye movements with third and sixth nerve palsies most commonly reported [11-23]. A good response to steroids is usually providing the diagnosis is made promptly. Our study has demonstrated that involvement of the eighth nerve is also not infrequently reported among patients with GCA. Deafness and tinnitus were the main symptoms reported by our patients and each occurred in over half of patients with GCA. These results are very similar to those reported in the one published prospective study in this area which showed similar prevalence figures for deafness and tinnitus [6]. This reported symptoms of unilateral or bilateral hearing loss, vertigo and tinnitus. Subjective hearing impairment was recorded in $64 \%$, $52 \%$ had vertigo and $50 \%$ had tinnitus. The symptoms of vestibular dysfunction were reversible in most cases following steroid therapy, while only $27 \%$ of GCA patients had hearing improvement after 3 months of steroids.

Although there have been several previous case reports and small series describing deafness and tinnitus in GCA [24-29], these amount to no more than 20 patients in total over nearly 50 years. Vertigo and dizziness have also been described in GCA in a small number of patients reported retrospectively [30]. The mechanism of vestibulocochlear involvement in giant cell arteritis is unclear but is thought to be secondary to inflammatory involvement of the vertebrobasilar system or the terminal cochleovestibular vessels [28]. The literature has not translated into a high level of awareness of this complication of the disease amongst most clinicians, but it appears that patients are much more aware of it. We suggest that clinicians need to be conscious of the association of eighth nerve dysfunction and GCA and that this may aid earlier diagnosis and prompt initiation of therapy with better patient-related outcomes. 
Table 3 To compare both the concurrence of vestibulocochlear symptoms with other symptoms and steroid responsiveness (among those with concurrence) between 128 patients with giant cell arteritis (GCA) and 189 with polymyalgia rheumatica (PMR) as numbers (percentages) of total

\begin{tabular}{|c|c|c|c|c|c|}
\hline & \multicolumn{2}{|c|}{ Symptom concurrence } & \multicolumn{2}{|c|}{ Steroid response } & \multirow[t]{2}{*}{$P$ values } \\
\hline & GCA & PMR & GCA & PMR & \\
\hline Hearing loss & $68(53 \%)$ & $23(12 \%)$ & $72(56 \%)$ & $9(5 \%)$ & $0.01,0.01$ \\
\hline Tinnitus & $43(33 \%)$ & $52(28 \%)$ & $54(42 \%)$ & $17(9 \%)$ & NS, 0.01 \\
\hline Loss of balance & $68(53 \%)$ & $86(45 \%)$ & $35(27 \%)$ & $32(17 \%)$ & NS, NS \\
\hline Vertigo & $64(50 \%)$ & $52(28 \%)$ & $22(17 \%)$ & $27(15 \%)$ & $0.04, \mathrm{NS}$ \\
\hline
\end{tabular}

Several studies have shown that GCA patients with elevated inflammatory markers have a lower risk of cranial ischaemic symptoms compared to those with lower inflammatory markers [31]. Pretreatment ESRs and CRP levels are inversely correlated with the risk of visual symptoms in GCA. One plausible explanation is that patients with cranial ischaemia present sooner, prior to developing a strong systemic inflammatory response [31]. Hence, awareness of the full range of symptoms suggesting possible intra-cranial involvement in GCA is essential if clinicians are to optimise their patients' outcome. Our data did not allow for differentiation between central and peripheral eighth nerve involvement. The presence of vertebrobasilar insufficiency and resulting cerebellar syndrome also increases with age and may have accounted for a significant percentage of vestibulocochlear symptomatology among our patients. Given that auditory acuity declines with age, the use of PMR patients as controls is essential in this study of patients with a mean age of 70 to account for such background pathology.

The marked difference in both the comparative rates between patients with GCA and PMR and in the responsiveness of these symptoms to steroid therapy in our study is therefore of great clinical interest. Clearly, features such as loss of balance and vertigo are less specific for eighth nerve dysfunction than is deafness and may have many other potential causes. Although both appeared more frequently among those with GCA, the reported responsiveness of these symptoms to steroid therapy was not as good as that of hearing loss, which is at odds with the prospective data [6].

The association between both colocation and concurrence of symptoms and subsequent steroid responsiveness is highly pertinent. Those patients whose symptoms of vestibulocochlear dysfunction were most likely to be directly related to their GCA diagnosis on the basis of their location and timing were nearly three times more likely to report an improvement in these symptoms on commencing steroid therapy. This emphasises the importance of a thorough history of this relationship to define the probability of such symptoms being directly attributable to the GCA and the relevance of these findings to the subsequent prognosis for recovery.
A major advantage of our study is its size. The literature consists mainly of scattered case reports so a substantial study across the UK constitutes a much larger evidence base to guide clinicians. The strong statistical association we report between symptoms of eighth nerve dysfunction and GCA should reinforce the importance of assessing symptoms of possible dysfunction in patients where GCA is suspected. The major limitation of our study is its retrospective nature and the fact that it relied entirely on patient recall as collected in questionnaire form. This approach is more likely to reduce rather than increase the estimated prevalence as elderly patients are more likely to forget symptoms rather than to invent them in this setting. We also accept that the lack of a physical examination to confirm or refute patient description of their symptoms is a further limitation of our study. However, given the wide geographic distribution of our patients included in the survey, this was the only practical way to contact the large numbers required for statistical significance. There is no reason to suggest this limitation could account for the significant observed differences between those patients with GCA and those with PMR. The similarity between our data and that reported in the only prospective study to date provides further reassurance about the likely accuracy of our retrospective data.

The dose of steroid used was much higher in those patients with GCA than those treated for PMR, as would be expected. We considered whether this could account for any of the observed increase in prevalence figures reported by patients with GCA as compared to those with PMR. This seemed to be highly unlikely as steroids are used to treat acute idiopathic hearing loss [32], which should therefore be less likely to persist in those patients who received higher doses of prednisone. However, it is interesting to speculate that such symptoms may also relate to an auto-immune vasculitis that could overlap and might even represent a limited form of GCA.

In summary, we report a high prevalence of self-recorded features of eighth nerve dysfunction among patients with GCA. Hearing loss and tinnitus are most strongly associated with other clinical features of the disease and are more likely to respond to the commencement of high-dose steroids. 
Funding This work was supported by the Charity 'PMR-GCA UK Support Group' and the results will be returned to them for dissemination amongst all their members.

\section{Compliance with ethical standards}

\section{Disclosures None.}

Open Access This article is distributed under the terms of the Creative Commons Attribution 4.0 International License (http:// creativecommons.org/licenses/by/4.0/), which permits unrestricted use, distribution, and reproduction in any medium, provided you give appropriate credit to the original author(s) and the source, provide a link to the Creative Commons license, and indicate if changes were made.

\section{References}

1. Yates M, Graham K, Arthur R et al (2016) The prevalence of giant cell arteritis and polymyalgia rheumatica in a UK primary care population. BMC Musculoskelet Disord 17:285-292

2. Salvarani C, Cantini F (2008) Hunder GG; Polymyalgia rheumatica and giant-cell arteritis. Lancet. 19372(9634):234-245

3. Hassan N, Dasgupta B, Barraclough K (2011) Giant cell arteritis. BMJ 342:233-232. https://doi.org/10.1136/bmj.d3019

4. Fytili C, Bournia VK, Korkou C, Pentazos G, Kokkinos A (2015) Multiple cranial nerve palsies in giant cell arteritis and response to cyclophosphamide: a case report and review of the literature. Rheumatol Int 35:773-776

5. Hausch RC, Harrington T (1998) Temporal arteritis and sensorineural hearing loss. Semin Arthritis Rheum 28:206-209

6. Amore-Dorado JC, Llorca J, Garcia-Porrua C, Costa C, PerezFernandez N, Gonzalez-Gay MA (2003) Audiovestibular manifestations in giant cell arteritis: a prospective study. Medicine (Baltimore) 82:13-26

7. Bernatsky S, Joseph L, Pineau C et al (2009) Polymyalgia rheumatica prevalence in a population-based sample. 27 August 2009. https://doi.org/10.1002/art.24793

8. Charlton R (2008 Oct) Polymyalgia rheumatica and its links with giant cell arteritis. Clin Med (Lond) 8(5):498-501

9. Levine DM, Stephan DF, Krehbiel TC, Berenson ML (2008) Statistics for managers: using Microsoft Excel, Fifth Edition. Pearson Education, Inc. Prentice Hall, Upper Saddle River, New Jersey

10. Park J, Junga M (2009) 'A note on determination of sample size for a Likert scale', Communications of the Korean Statistical Society [Online]16(4) pp: 669-673, available from:

11. Loffredo L, Parrotto F, Violi F (2004) Giant cell arteritis, oculomotor nerve palsy, and acute hearing loss. Scandinavian Journal of Rheumatology 33(4):279-280. https://doi.org/10.1080/ 03009740410006178

12. Bondeson J, Asman P (1997) Giant cell arteritis presenting with oculomotor nerve palsy. Scand J Rheumatol 26:327-328

13. Davies GE, Shakir RA (1994) Giant cell arteritis presenting as oculomotor nerve palsy with pupillary dilatation. Postgrad Med J 70:298-299
14. Hayreh SS, Podhajsky PA, Zimmerman B (1998) Ocular manifestations of giant cell arteritis. Am J Ophthalmol 125:509-520

15. Fisher CM (1959) Ocular palsy in temporal arteritis. Minn Med 42: $1258-1268$

16. Biousse V, Newman NJ (2000) Third nerve palsy. Semin Neurol 20: $55-74$

17. Thurtell MJ, Longmuir RA (2014) Third nerve palsy as the initial manifestation of giant cell arteritis. J Neuroophthalmol 0:1-3. https://doi.org/10.1097/WNO.0000000000000116

18. Quinn R, Hawkes C, Lodhi A et al (2018) Ophthalmoplegia in an elderly woman with giant cell arteritis. Rheumatol Adv Pract 2(2): rky020. https://doi.org/10.1093/rap/rky020

19. Chew SSL, Kerr NM, Danesh-Meyer HV (2009) Giant cell arteritis. J Clin Neurosci 16:1263-1268

20. Gaudre N, Michaud M, Soler V, Lozano S, Gaudin C (2015) Balardy LG6: Giant cell arteritis: a reversible cause of oculomotor nerve palsy. Presse Med 44:859-860

21. Mehler MF, Rabinowich L (1988) The clinical neuroophthalmologic spectrum of temporal arteritis. Am J Med 85: 839-844

22. Li LM, Khor GT, Lan SH, Lai CL (2007) Giant cell arteritis with multiple cranial nerve palsy and reversible proptosis: a case report. Headache. 47:1451-1453

23. Foroozan R, Deramo VA, Buono LM, Jayamanne DGR, Sergott RC, Danesh-Meyer H, Savino PJ (2003) Recovery of visual function in patients with biopsy-proven giant cell arteritis. Ophthalmology. 110:539-542

24. Berrettini S, Ravecca F, Bruschini L, Ursino F, Sellari-Franceschini S (1998) Progressive sensorineural hearing loss: immunological causes. Acta Otorhinolaryngol Ital 59:S33-S42

25. Le N, Vickers A, Ponce C, Chevez-Barrios P, Lee A (2019) Vestibulocochlear symptoms as the initial presentation of giant cell arteritis. Canadian J Opthalmol 54(1):e1-e3

26. Rauch SD (2008) Clinical practice. Idiopathic sudden sensorineural hearing loss. N Engl J Med 359:833-840

27. Francis DA, Boddie HG (1982) Acute hearing loss in giant cell arteritis. Postgrad Med J 58:357-358

28. Junejo S, Ali Y, Abrudescu A (2017) Giant cell (temporal) arteritis with persistent bilateral sensorineural hearing loss - a likely consequence of delayed institution of glucocorticoid therapy. Am J Case Rep 18:1233-1237. https://doi.org/10.12659/AJCR.907174

29. Saadoun D, Cacoub P, Costedoat-Chalumeau N, Sbai A, Piette JC (2000) Horton's disease and corticosteroid-responsive hearing loss. Ann Med Int 151:600-602

30. McKennan KX, Nielsen SL, Watson C, Wiesner K (1993) Meniere's syndrome: an atypical presentation of giant cell arteritis (temporal arteritis). Laryngoscope. 103:1103-1107

31. Cid MC, Font C, Oristrell J et al (1998) Association between strong inflammatory response and low risk of developing visual loss and other cranial ischemic complications in giant cell (temporal) arteritis. Arthritis Rheumatol 41(1):26-32. https://doi.org/10.1002/ 1529-0131(199801)41:1<26::AID-ART4>3.0.CO;2-0

32. Goodall AF (2015 Apr 6) Current understanding of the pathogenesis of autoimmune inner ear disease: a review. Clin Otolaryngol 40:412-419. https://doi.org/10.1111/coa. 12432

Publisher's note Springer Nature remains neutral with regard to jurisdictional claims in published maps and institutional affiliations. 